\title{
ESTUDO DA DEGRADAÇÃO E MINERALIZAÇÃO DE UM EFLUENTE LÍQUIDO FENÓLICO POR OXIDAÇÃO AVANÇADA EM REATOR BATELDA LABORATÓRIAL
}

\author{
B. A. AMARAL ${ }^{1}$, J. R. TEODOSIO ${ }^{1}$, Y. B. BRANDÃO ${ }^{1,2}$ e M. BENACHOUR ${ }^{1}$ \\ ${ }^{1}$ Universidade Federal de Pernambuco, Departamento de Engenharia Química \\ ${ }^{2}$ Universidade Católica de Pernambuco, Departamento de Engenharia Química \\ E-mail: bruno_de_albuquerque@hotmail.com
}

\begin{abstract}
RESUMO - O objetivo deste trabalho é quantificar o efeito da Temperatura (T), da Razão Molar Estequiométrica Fenol/Peróxido de Hidrogênio (R), da Vazão de Ar $\left(\mathrm{Q}_{\mathrm{AR}}\right)$ e do $\mathrm{pH}$ do efluente no tratamento por Processo de Oxidação Avançada (POA) de um efluente líquido fenólico. Neste processo, o peróxido de hidrogênio foi utilizado como fonte de radical hidroxila, eficaz na degradação do composto orgânico. Os estudos experimentais foram realizados em um reator batelada em escala laboratorial, tendo um efluente fenólico sintético com uma concentração inicial de $500 \mathrm{mg} \cdot \mathrm{L}^{-1}$. A degradação do fenol foi acompanhada com o uso da técnica analítica da Cromatografia Líquida de Alta Eficiência (CLAE) e a mineralização do composto foi monitorada com um analisador de Carbono Orgânico Total (COT). Uma degradação do fenol superior a $98 \%$ e uma conversão do COT superior a 56\% foram obtidas, respectivamente, nas condições operacionais ótimas do processo $\left(\mathrm{T}=90{ }^{0} \mathrm{C} ; \mathrm{R}=100 \%, \mathrm{pH}=7\right.$ e $\left.\mathrm{Q}_{\mathrm{AR}}=100 \mathrm{NL} \cdot \mathrm{h}^{-1}\right)$.
\end{abstract}

\section{INTRODUÇÃO}

O fenol é um composto tóxico, solúvel em água e em solventes orgânicos e com odor característico que pode causar sérios problemas à saúde humana e ao meio ambiente, mesmo em concentrações na faixa de mg. $\mathrm{L}^{-1}$ (Benali e Guy, 2007; Brito e Rangel, 2008). Este composto está presente em diversos tipos de efluentes industriais, tais como: farmacêutica, pesticidas, nas fábricas de resina, plásticos, corantes e refinarias de petróleo dentre outros (Rodrigues et al., 2010; Aghav et al., 2011).

Devido ao risco que os efluentes fenólicos apresentam é necessário utilizar algumas formas para tratar estes efluentes. Vários métodos podem ser utilizados para a remoção dos compostos fenólicos, como por exemplo, a adsorção, troca de íons, osmose reversa, precipitação e outros (Brandão et al., 2010; Aghav et al., 2011). Os tratamentos de efluentes podem ser divididos em três tipos: os tratamentos físicos, químicos e biológicos. Uma combinação destes três tratamentos é geralmente utilizada (Matta et al., 2008).

Um método para tratamento de efluentes contaminados é o Processo Oxidativo Avançado 


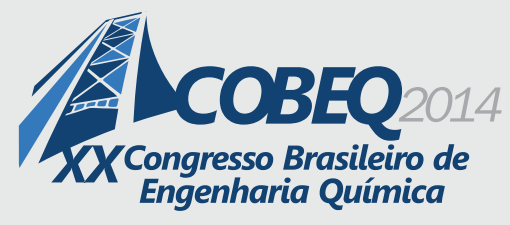

19 a 22 de outubro de 2014
Florianópolis/SC

(POA) devido à formação de radicais hidroxila $(\bullet \mathrm{OH})$ quando utilizado isoladamente ou combinados com agentes oxidantes, tais como: o oxigênio, peróxido de hidrogênio, ozônio e outros. Por serem altamente oxidantes tem a capacidade de mineralizar a matéria orgânica transformando esta em dióxido de carbono, água e íons inorgânicos (Teixeira e Jardim, 2004). O POA tem se destacado entre os outros tipos de tratamentos de efluentes por não necessitar de altas temperaturas e pressões, além de apresentar um custo operacional baixo. Além disso, o POA apresenta uma alta eficiência na mineralização de compostos orgânicos, sendo assim um ótimo tipo de tratamento para efluentes contaminados (Brito e Rangel, 2008).

Este trabalho tem como objetivo estudar o uso de um POA no tratamento de um efluente sintético contendo fenol, a fim de determinar a degradação do fenol e a conversão do Carbono Orgânico Total (COT). Os estudos experimentais foram realizados, para avaliar o efeito da Temperatura (T), da Razão Molar Estequiométrica Fenol/Peróxido de Hidrogênio (R), do pH e da Vazão de $\operatorname{Ar}\left(\mathrm{Q}_{\mathrm{AR}}\right)$ sobre a etapa de degradação do fenol utilizando o método da Cromatografia Líquida de Alta Eficiência (CLAE) e da conversão do COT usando um analisador de COT.

\section{MATERIAIS E MÉTODOS}

\subsection{Reator de Bancada Tipo PARR}

O reator de bancada PARR foi utilizado na oxidação das amostras do efluente fenólico. Para cada um dos 28 ensaios, foi preparado um efluente contendo fenol com uma concentração aproximada de 500 mg. $\mathrm{L}^{-1}$, a fim de avaliar o efeito dos parâmetros operacionais sobre a eficiência do processo. $\mathrm{O}$ volume do efluente preparado para todos os experimentos foi de 1,3 L. O controle do $\mathrm{pH}$ do efluente foi realizado com o auxilio de um $\mathrm{pH}$-metro, adicionando ácido ou base a solução até atingir o $\mathrm{pH}$ desejado para realizar a análise. Após preparar o efluente sintético, o composto fenólico foi adicionado no reator PARR. O controle da temperatura do efluente foi monitorado no painel de controle do reator, onde também pode ser controlada a rotação do agitador e a pressão do sistema, sendo utilizada a pressão atmosférica. Após a estabilizar a temperatura do efluente, foi coletada uma amostra e em seguida adicionado o peróxido de hidrogênio ao efluente e ajustado a vazão de ar, dando início ao processo de degradação do fenol e da conversão do COT ao longo do tempo operacional. O efluente restante foi então diluído, quando necessário, em níveis de concentração, conforme a resolução ambiental de descarte $n^{\circ} 430$ de 2011 do CONAMA (Conama, 2011). As análises quantitativas dos níveis de concentração de descarte do resíduo de fenol e intermediários são realizadas através da técnica de CLAE, imediatamente após coleta e resfriamento da amostra. A Figura 1 mostra um esquema sucinto do reator utilizado nos ensaios com a oxidação do efluente orgânico fenólico. Em todos os ensaios experimentais de oxidação do fenol, totalizando 28, foram coletadas amostras ao longo do tempo operacional, sendo observada uma mudança na coloração até atingir cor praticamente preta, dependendo do nível de degradação da amostra, evidenciando que ocorreu de fato a oxidação do fenol. Em seguida, as amostras foram resfriadas, filtradas (papel de filtro de $2 \mu \mathrm{m}$ ) e posterior medição do valor de $\mathrm{pH}$ e análise por técnicas de CLAE e COT. 


\section{9 a 22 de outubro de 2014 \\ Florianópolis/SC}

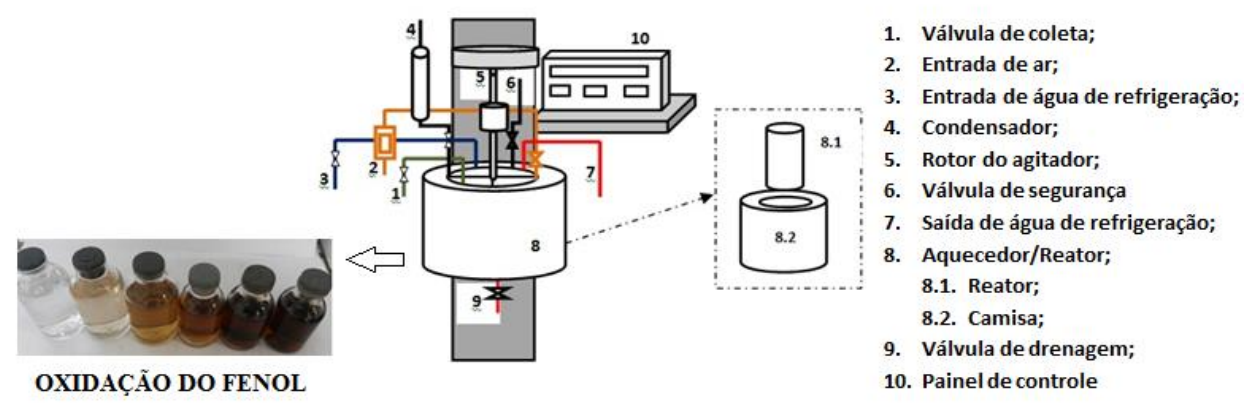

Figura 1 - Esquema sucinto do reator batelada tipo PARR.

\subsection{Métodos Analíticos}

Cromatografia líquida de alta eficiência: Para análise cromatográfica da concentração residual do fenol, as amostras foram analisadas no Cromatógrafo CLAE (modelo LC 20AT - Shimadzu), com detecção UV-VIS, degaseificador (DGU 20As), módulo (CBM 20A), forno (CTO-20A) com sistema integrado de aquisição de dados, utilizando uma coluna $(\mathrm{M}) /(\mathrm{C}-18)$ de $25 \mathrm{~cm}$. O modo de eluição foi o isocrático, temperatura do forno $\left(35^{0} \mathrm{C}\right)$, volume de injeção $(20 \mu \mathrm{L})$, composição da fase móvel (10\% metanol e $90 \%$ água/ácido fosfórico com um pH ajustado para 2,2), vazão da fase móvel $(0,75$ mL. $\min ^{-1}$ ) e comprimento de onda do detector UV de $270 \mathrm{~nm}$ para identificar o fenol. Os reagentes utilizados foram: fenol (99\% P.A., marca DINÂMICA), metanol UV/HPLC (98,5\% P.A., marca DINÂMICA) e ácido fosfórico UV/HPLC (85\% P.A., marca DINÂMICA).

Analisador de carbono orgânico total: As determinações de Carbono Orgânico Total (COT)

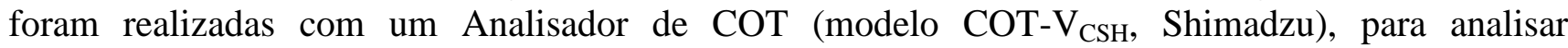
quantitativamente. O COT é a subtração entre o teor de Carbono Total (CT) e Carbono Inorgânico (CI) (Fonseca et al., 2006).

\subsection{Grandezas Calculadas}

A Razão Molar Estequiométrica de Fenol/Peróxido de Hidrogênio (R) de 100\% corresponde à completa mineralização de fenol de acordo com a estequiometria da reação (Equação 1):

$$
\mathrm{C}_{6} \mathrm{H}_{5} \mathrm{OH}+14 \mathrm{H}_{2} \mathrm{O}_{2} \rightarrow 6 \mathrm{CO}_{2}+17 \mathrm{H}_{2} \mathrm{O}
$$

Razões molares de $\mathrm{R}$ (diferentes de 100\%) são calculadas proporcionalmente conforme previsto pela estequiometria da reação acima.

A degradação do fenol ( $\left.\mathrm{X}_{\mathrm{DF}}\right)$, em \%, foi avaliada de acordo com a Equação 2:

$$
X_{D F}=\frac{C_{F 0}-C_{F}}{C_{F 0}}
$$


Sendo o $\mathrm{C}_{\mathrm{F}_{0}} \mathrm{O}$ valor da concentração inicial do fenol e $\mathrm{C}_{\mathrm{F}}$ a concentração do fenol no instante t.

A conversão do COT ( $\left.\mathrm{X}_{\mathrm{COT}}\right)$, em \%, foi quantificada conforme a Equação 3:

$$
X_{C O T}=\frac{C O T^{0}-C O T}{C O T^{0}-C O T_{B}}
$$
o efluente.

Sendo o $\mathrm{COT}^{0}$ o valor do COT para $\mathrm{t}=0$ e $\operatorname{COT}_{B}$ o valor do COT da água utilizada para preparar

\subsection{RESULTADOS E DISCUSSÃO}

Neste trabalho foi utilizado um planejamento experimental do tipo Delineamento Composto Central Rotacional - DCCR, com quatro variáveis estudadas: Temperatura (T), Razão Molar Fenol/Peróxido de Hidrogênio $(\mathrm{R})$, potencial Hidrogeniônico $(\mathrm{pH})$ do efluente e vazão de ar $\left(\mathrm{Q}_{\mathrm{AR}}\right)$. A Tabela 1 apresenta os valores dos níveis das variáveis utilizadas no planejamento DCCR.

Tabela 1 - Variáveis operacionais do processo oxidativo avançado

\begin{tabular}{l|c|c|c|c|c}
\hline Fatores & Nível (-2) & Nível (-1) & Ponto Central (0) & Nível (+1) & Nível (+2) \\
\hline $\mathrm{pH}$ & 3 & 4 & 7 & 10 & 11 \\
Temperatura $\left({ }^{\circ} \mathrm{C}\right)$ & 50 & 60 & 70 & 80 & 90 \\
Razão fenol $/ \mathrm{H}_{2} \mathrm{O}_{2}(\%)$ & 0 & 25 & 50 & 75 & 100 \\
Vazão de $\mathrm{Ar}\left(\mathrm{NL} \mathrm{h}^{-1}\right)$ & 0 & 50 & 100 & 150 & 200 \\
\hline
\end{tabular}

A Tabela 2 apresenta a matriz do planejamento com os resultados obtidos para a degradação do fenol $\left(\mathrm{X}_{\mathrm{DF}}\right)$ e conversão do Carbono Orgânico Total - COT ( $\left.\mathrm{X}_{\mathrm{COT}}\right)$.

As significâncias dos efeitos principais e das interações das variáveis para o planejamento em relação à degradação do fenol e conversão do COT estão representadas na Figura 2, onde (L) representa a parte linear e (Q) a parte quadrática do modelo. Os valores que ultrapassam a linha $\mathrm{p}=0,05$ são aqueles que foram significativos para o intervalo de confiança de $95 \%$.

Apenas a temperatura $(T)$ e a razão molar $(\mathrm{R})$, quadráticas $(\mathrm{Q})$, e, a interação entre a razão molar (R) e a vazão de ar $\left(\mathrm{Q}_{\mathrm{AR}}\right)$, Linear (L), não foram significativas para a conversão de COT. Enquanto, a temperatura $(T)$ quadrática $(\mathrm{Q})$ e a interação entre a temperatura $(T)$ e vazão de $\operatorname{ar}\left(\mathrm{Q}_{\mathrm{AR}}\right)$, Linear (L), não foram significativas para a degradação do fenol. A análise de variância para o planejamento, para $\mathrm{X}_{\mathrm{DF}}$ e $\mathrm{X}_{\mathrm{COT}}$, esta representada na Tabela 3. O valor do F tabelado, para $\mathrm{X}_{\mathrm{DF}} \mathrm{e}$ $\mathrm{X}_{\mathrm{COT}}$, de acordo com o grau de liberdade é de: $\mathrm{F}_{\text {tab }}(14 ; 13 ; 0,05)=2,67$. 


\section{ACOBEQ

Tabela 2 - Matriz planejamento e média dos valores de degradação do fenol e conversão do COT.

\begin{tabular}{|c|c|c|c|c|c|c|c|c|c|c|c|c|c|}
\hline Ensaio & pH & $\begin{array}{c}\mathbf{T} \\
\left({ }^{\circ} \mathrm{C}\right)\end{array}$ & $\begin{array}{c}\mathbf{R} \\
(\%) \\
\end{array}$ & $\begin{array}{c}\mathbf{Q}_{\mathrm{AR}} \\
(\mathrm{NL} . \\
\left.\mathbf{h}^{-1}\right)\end{array}$ & $\begin{array}{l}\mathbf{X}_{\mathrm{DF}} \\
(\%) \\
\end{array}$ & $\begin{array}{c}\mathbf{X}_{\mathbf{C O T}} \\
(\%)\end{array}$ & Ensaio & pH & $\begin{array}{c}\mathbf{T} \\
\left({ }^{\circ} \mathbf{C}\right)\end{array}$ & $\begin{array}{c}\mathrm{R} \\
(\%)\end{array}$ & $\begin{array}{c}\mathbf{Q}_{\mathrm{AR}} \\
(\mathrm{NL} . \\
\left.\mathbf{h}^{-1}\right)\end{array}$ & $\begin{array}{l}X_{\mathrm{DF}} \\
(\%)\end{array}$ & $\begin{array}{c}X_{\text {COT }} \\
(\%)\end{array}$ \\
\hline 1 & -1 & -1 & -1 & -1 & 63,7 & 5,8 & 15 & +1 & +1 & +1 & -1 & 21,8 & 9,8 \\
\hline 2 & -1 & -1 & -1 & +1 & 62,2 & 2,8 & 16 & +1 & +1 & +1 & +1 & 37,8 & 17,3 \\
\hline 3 & -1 & -1 & +1 & -1 & 62,9 & 10,5 & 17 & 0 & 0 & 0 & 0 & 83,1 & 15 \\
\hline 4 & -1 & -1 & +1 & +1 & 43,8 & 6,3 & 18 & 0 & 0 & 0 & 0 & 82,8 & 14,9 \\
\hline 5 & -1 & +1 & -1 & -1 & 68,3 & 7 & 19 & 0 & 0 & 0 & 0 & 82,1 & 13,5 \\
\hline 6 & -1 & +1 & -1 & +1 & 62,2 & 13,6 & 20 & 0 & 0 & 0 & 0 & 84 & 15,2 \\
\hline 7 & -1 & +1 & +1 & -1 & 81,4 & 21,1 & 21 & -2 & 0 & 0 & 0 & 11,8 & 5,4 \\
\hline 8 & -1 & +1 & +1 & +1 & 97,3 & 28,6 & 22 & +2 & 0 & 0 & 0 & 15,2 & 3,1 \\
\hline 9 & +1 & -1 & -1 & -1 & 2,9 & 1,3 & 23 & 0 & -2 & 0 & 0 & 42,1 & 5,2 \\
\hline 10 & +1 & -1 & -1 & +1 & 9,6 & 2,5 & 24 & 0 & +2 & 0 & 0 & 91,2 & 22,2 \\
\hline 11 & +1 & -1 & +1 & -1 & 11,9 & 3,5 & 25 & 0 & 0 & -2 & 0 & 2,2 & 4,8 \\
\hline 12 & +1 & -1 & +1 & +1 & 46,2 & 5,7 & 26 & 0 & 0 & +2 & 0 & 81,2 & 17,8 \\
\hline 13 & +1 & +1 & -1 & -1 & 13,5 & 4,7 & 27 & 0 & 0 & 0 & -2 & 27,2 & 2,7 \\
\hline 14 & +1 & +1 & -1 & +1 & 16,6 & 11,4 & 28 & 0 & 0 & 0 & +2 & 60,8 & 17,8 \\
\hline
\end{tabular}
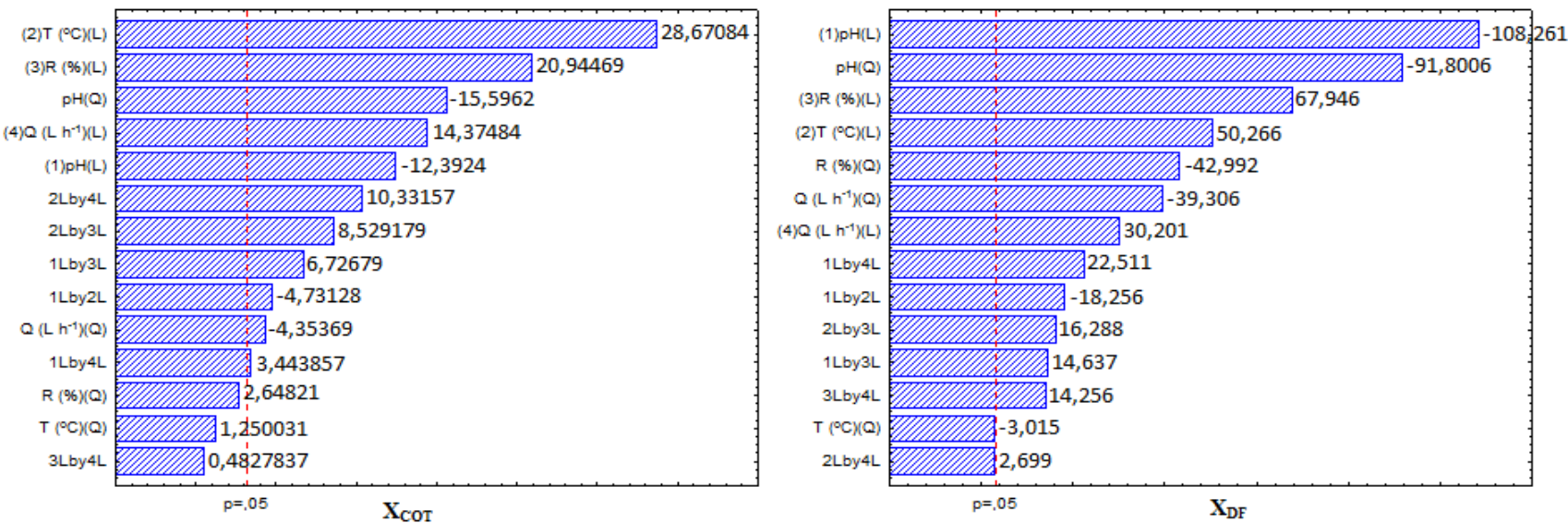

Figura 2 - Gráfico de Pareto do planejamento fatorial do tipo DCCR para $\mathrm{X}_{\mathrm{DF}}$ e $\mathrm{X}_{\mathrm{COT}}$.

Tabela- 3 Análise de variância do planejamento para $\mathrm{X}_{\mathrm{DF}}$ e $\mathrm{X}_{\mathrm{COT}}$.

\begin{tabular}{|c|c|c|c|c|c|c|c|c|}
\hline \multirow{2}{*}{$\begin{array}{l}\text { Fonte de } \\
\text { Variação }\end{array}$} & \multicolumn{2}{|c|}{ Soma Quadrática } & \multicolumn{2}{|c|}{$\mathrm{N}^{0}$ de g. 1.} & \multicolumn{2}{|c|}{ Média quadrática } & \multicolumn{2}{|c|}{ Fcalculado $(p \leq 0,05)$} \\
\hline & $\overline{\mathbf{X}_{\text {COT }}}$ & $\mathbf{X}_{\mathrm{DF}}$ & $\overline{X_{\text {COT }}}$ & $\overline{\mathbf{X}_{\mathrm{DF}}}$ & $\overline{\mathbf{X}_{\mathrm{COT}}}$ & $\mathbf{X}_{\mathrm{DF}}$ & $\overline{X_{\text {COT }}}$ & $\overline{\mathbf{X}_{\mathrm{DF}}}$ \\
\hline Regressão & 1294,44 & 19832,4 & 14 & 14 & 92,46 & 1416,6 & 14,32 & 3,19 \\
\hline Resíduos & 83,92 & 5765,46 & 13 & 13 & 6,46 & 443,5 & & \\
\hline Total & 1378,35 & 25597,9 & 27 & 27 & & & & \\
\hline
\end{tabular}


Pelo teste $\mathrm{F}$, tem-se que o $\mathrm{F}_{\text {calc }}>\mathrm{F}_{\text {tab}}$, portanto, o modelo quadrático é considerado válido para a degradação do fenol e conversão do COT, logo, descreve satisfatoriamente a superfície de resposta. Com os resultados obtidos no planejamento do tipo DCCR, foi possível identificar as condições ótimas com o auxilio do software STATISTICA versão 8.0, sendo estas as seguintes: temperatura $\mathrm{T}=90^{\circ} \mathrm{C}$, razão molar $\mathrm{R}=100 \%, \mathrm{pH}=7$ e vazão de $\operatorname{ar} \mathrm{Q}_{\mathrm{G}}=100 \mathrm{NL} \cdot \mathrm{h}^{-1}$.

Um experimento foi realizado em triplicata nas condições ótimas onde foi obtida a média aritmética dos valores de degradação do fenol de $98 \%$ e a média da conversão do COT de 56\%. Nesta etapa, as análises do COT foram realizadas em intervalos de tempo menores, com objetivo de elaborar um modelo cinético para a conversão do COT. A Figura 3 representa os resultados do perfil temporal do COT e do $\mathrm{pH}$ do líquido presente no reator PARR, nas condições operacionais ótimas do processo.

Analisando os dados da Figura 3 foi observado que ao longo do processo o $\mathrm{pH}$ do meio diminui até ficar constante (igual a 3). Isso ocorre devido à formação de ácidos durante o processo de oxidação, onde o fenol é degradado formando intermediários aromáticos, tais como o catecol e a hidroquinona, e em seguida estes são degradados dando origem a diversos tipos de ácidos, até que sejam convertidos em $\mathrm{CO}_{2}$ e $\mathrm{H}_{2} \mathrm{O}$ (Devlin e Harris, 1984). A partir desta redução do $\mathrm{pH}$ pode ser concluído, que em 10 minutos de processo uma parte do fenol e de seus intermediários já foi degradada, dando origem a diversos tipos de ácidos.
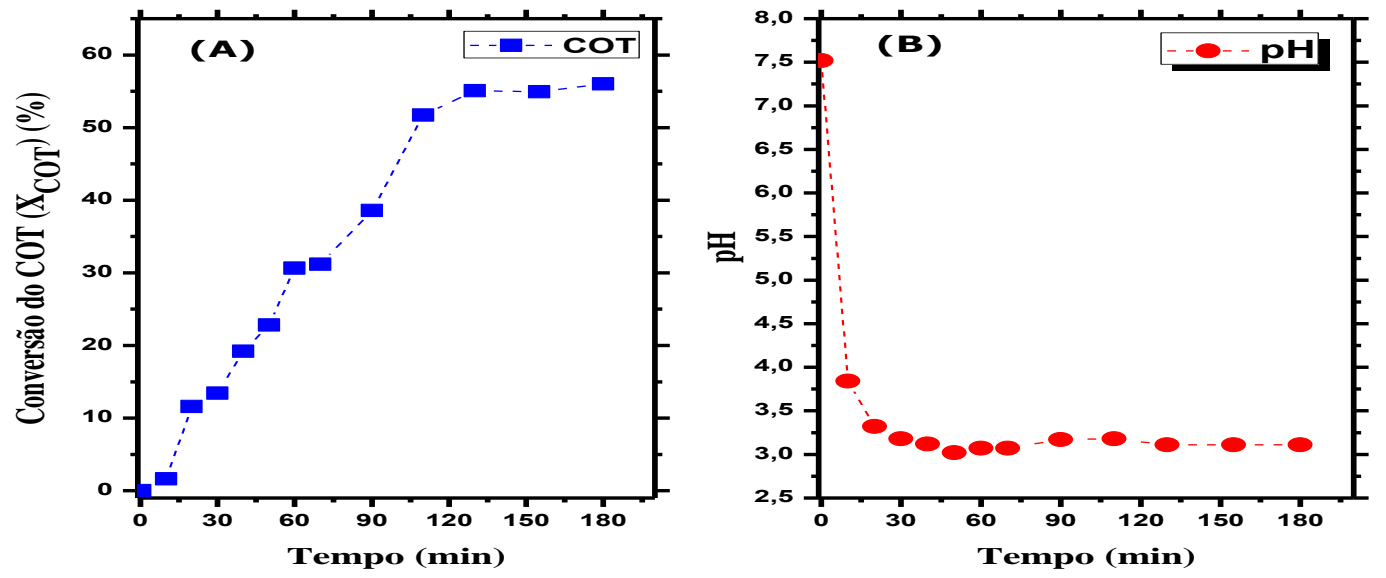

Figura 3 - Evolução da: A) Conversão do COT e B) pH, respectivamente, em função do tempo. $\left(\mathrm{T}=90{ }^{0} \mathrm{C} ; \mathrm{R}=100 \%\right.$ e $\left.\mathrm{Q}_{\mathrm{AR}}=100 \mathrm{NL} \cdot \mathrm{h}^{-1}\right)$.

A conversão do COT pode ocorrer por dois caminhos diferentes de acordo com o modelo cinético proposto por Li (1991) conforme mostra a Figura 4. Nesta, a espécie (A) designa o grupo de carbono do fenol e dos compostos intermediários não refratários que podem ser degradado de maneira direta dando origem a $\mathrm{CO}_{2} \mathrm{e}_{2} \mathrm{O}$ (espécies $\mathrm{C}$ ), como também pode formar um grupo de carbono dos compostos intermediários refratários (espécie $\mathrm{B}$ ), e esses intermediários podem ser convertidos por sua vez em $\mathrm{CO}_{2}$ e $\mathrm{H}_{2} \mathrm{O}$ (espécies $\mathrm{C}$ ). As constantes $\mathrm{k}_{1}, \mathrm{k}_{2}$ e $\mathrm{k}_{3}$ representam as constantes de velocidade para cada uma dessas reações consideradas de primeira ordem, sendo ao tempo $t=0, \mathrm{C}_{\mathrm{A}}=\mathrm{C}_{\mathrm{AO}}=\mathrm{COT}_{0} \mathrm{e}$ $\mathrm{C}_{\mathrm{B}}=\mathrm{C}_{\mathrm{C}}=0$. 


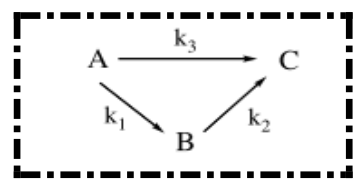

Figura 4 - Reações para conversão do COT.

A Equação 4 representa o modele cinético para a conversão do COT. Os valores de suas constantes foram encontrados com o uso de uma ferramenta do Excel chamada "Solver" e com os resultados obtidos da conversão do COT:

$\frac{C_{A}+C_{B}}{C_{A}^{o}}=\frac{C O T}{C O T^{o}}=\frac{k_{1}}{k_{1}+k_{3}-k_{2}} e^{-k_{2} t}+\frac{k_{3}-k_{2}}{k_{1}+k_{3}-k_{2}} e^{-\left(k_{1}+k_{3}\right) t}$

Os valores obtidos para as constantes $\mathrm{k}_{1}, \mathrm{k}_{2}$ e $\mathrm{k}_{3}$ foram 1,730 $\min ^{-1}, 0,005 \mathrm{~min}^{-1}$ e 0,013 $\min ^{-1}$, respectivamente.. O elevado valor encontrado para o $\mathrm{k}_{1}$ justifica a redução rápida do $\mathrm{pH}$ no meio, indicando que a degradação do fenol formando intermediários ocorreu rapidamente. Os valores do $\mathrm{COT} / \mathrm{COT}^{0}$ experimentais e calculados estão representados na Figura 5 considerando o modelo cinético proposto. Nesta, foi verificado que os valores calculados pelo modelo cinético proposto estão próximos aos experimentais.

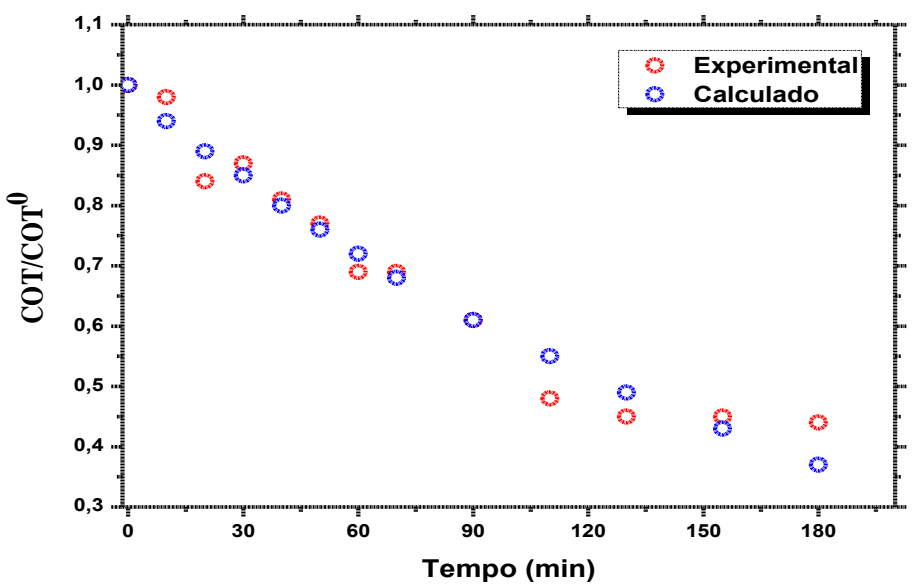

Figura 5 - Valores do COT/COT ${ }^{0}$ calculados e experimentais ao longo do tempo

\section{CONCLUSÃO}

Neste trabalho foi possível observar que todas as variáveis operacionais foram significativas dentro do intervalo estudado. Para o efeito da razão molar fenol/peróxido ( $R$ ), foi confirmado que a presença de um agente oxidante é fundamental para que ocorra o processo de oxidação do composto orgânico. No ensaio 25 em que não teve a adição do peróxido de hidrogênio, os níveis de degradação do fenol e conversão do COT foram baixos, devido à ausência de um agente oxidante responsável pela formação dos radicais hidroxila. Foi possível observar também, que com o aumento da Temperatura (T) do meio, o processo de oxidação foi favorecido, alcançando valores maiores na 
degradação do fenol e na conversão do COT. No ensaio realizado com as condições otimizadas do processo $\left(\mathrm{T}=90{ }^{0} \mathrm{C} ; \mathrm{R}=100 \%, \mathrm{pH}=7\right.$ e $\left.\mathrm{Q}_{\mathrm{AR}}=100 \mathrm{NL} \cdot \mathrm{h}^{-1}\right)$, a degradação do fenol e a conversão do COT alcançaram valores mais elevados de $98 \%$ e $56 \%$, respectivamente, sendo mais significativo para a conversão do COT. Nesta etapa, foi possível observar que a oxidação do fenol começa a ocorrer com pelo menos 10 minutos de reação, devido a redução de $\mathrm{pH}$ do meio, pela formação de ácidos a partir da oxidação do fenol. $\mathrm{O}$ valor relativamente elevado da constante de velocidade $\mathrm{k}_{1}$ para o modelo cinético proposto comprova que a degradação do fenol ocorreu mais rapidamente.

\section{BIBLIOGRAFIA}

AGHAV, R. M.; KUMAR, S.; MUKHERJEE, S. N. Artificial neural network modeling in competitive adsorption of phenol and resorcinol from water environment using some carbonaceous adsorbents. J. HAZARD. MATER. v. 188, n. 1-3, p. 67-77, 2011.

BENALI, M.; GUY, C. Thermochemical oxidation of phenolic-laden liquid effluent models. $J$. Environ. Eng. Sci., v. 6, n. 5, p. 543-552, 2007.

BRANDÃO, Y.; TEODOSIO, J.; BENACHOUR, M.; OLIVEIRA, J.; MARINHO, I.; FIGUEIRÊDO, F.; ANSELMO-FILHO, P. Estudo do efeito do excesso de ar e da potência dissipada do queimador sobre as capacidades do processo DiCTT no tratamento de efluentes líquidos fenólicos. Rev. Iberoam. Sist. Cib. Inf. 7:1-9, 2010.

BRITTO, J. M.; RANGEL, M. C. Processos avançados de oxidação de compostos fenólicos em efluentes industriais. Quim. Nova., v. 31, n. 1, p. 114-122, 2008.

Conselho Nacional de Meio Ambiente (CONAMA). Resolução n. 43; 2011.

DEVLIN, H. R.; HARRIS, I. J. Mechanism of the oxidation of aqueous phenol with dissolved oxygen. Ind. Eng. Chem. Res. v. 233, p. 87-92, 1984.

FONSECA, J. C. L.; SILVA, M. R. A.; BAUTITZ, I. R.; NOGUEIRA, R. F. P.; MARCHI, M. R. R. Avaliação da confiabilidade analítica das determinações de carbono orgânico total (COT). Ecletica. Quim. 31:47-52, 2006.

LI, L.; CHEN, P.; GLKOYNA, E. F. Generalized kinetic model for wet oxidation of orgânic compounds. AIChE J., v.11, n 37, p. 1687-1697, 1991.

MATTA, R.; HANNA, K.; CHIRON, S. Oxidation of phenol by green rust and hydrogen peroxide at neutral pH. Sep. Purif. Technol. 61:442-6, 2008.

RODRIGUES, G. D., SILVA, L. H. M, SILVA, M. C. H. Alternativas verdes para o preparo de amostra e determinação de poluentes fenólicos em água. Quim. Nova. v. 33, n. 6, p. 1370-1378, 2010.

TEIXEIRA, C. P. A. B.; JARDIM, W. F. Caderno Temático: Processos oxidativos avançados Conceitos teóricos. Universidade Estadual de Campinas, v. 3. Campinas: UNICAMP, 2004. 